\title{
Histomorphometric assessment of bone necrosis produced by two cryosurgery protocols using liquid nitrogen: an experimental study on rat femurs
}

\author{
Fábio Wildson Gurgel COSTA', Gerly Anne de Castro BRITO², Rosana Maria Andrade PESSOA ${ }^{3}$, Eduardo Costa \\ STUDART-SOARES ${ }^{4}$
}

\begin{abstract}
1- DDS, MSc, Assistant Professor, Division of Stomatology, Department of Clinical Dentistry, Federal University of Ceará, Sobral, CE, Brazil. 2- MD, MSc, PhD, Associate Professor, Division of Histology, Department of Physiology and Pharmacology, School of Medicine, Federal University of Ceará, Fortaleza, CE, Brazil.

3- DDS, Graduate student, Division of Oral Surgery, Department of Clinical Dentistry, Federal University of Ceará, Fortaleza, CE, Brazil.

4- DDS, MSc, PhD, Associate Professor, Division of Oral Surgery, Department of Clinical Dentistry, Federal University of Ceará, Fortaleza, CE, Brazil.
\end{abstract}

Corresponding address: Fábio Wildson Gurgel Costa - Coordenação do Curso de Odontologia da Universidade Federal do Ceará - Campus Sobral - Av. - Comte. Maurocélio Rocha Pontes, s/nº Derby - 62041-040 - Sobral - Ceará - Brazil - Phone: +55 (88) 36132603 - Fax: +55 (88) 36132603 - e-mail: fwildson@yahoo.com.br

Received: September 24, 2009 - Accepted: April 30, 2010

\section{ABSTRACT}

\begin{abstract}
bjective: The aim of this study was to evaluate the effects of liquid nitrogen cryosurgery on the femoral diaphysis of rats. Material and Methods: The femoral diaphyses of 42 Wistar rats were exposed to three local and sequential applications of liquid nitrogen for 1 or $2 \mathrm{~min}$, intercalated with periods of $5 \mathrm{~min}$ of passive thawing. The animals were sacrificed after 1, 2, 4 and 12 weeks and the specimens obtained were processed and analyzed histomorphometrically. Results: The depth and extent of peak bone necrosis were 124.509 $\mu \mathrm{m}$ and $2087.094 \mu \mathrm{m}$ for the 1-min protocol, respectively, and 436.424 $\mu \mathrm{m}$ and 12046.426 $\mu \mathrm{m}$ for the 2-min protocol. Peak necrosis was observed in the second experimental week with both cryotherapy protocols. Conclusions: The present results indicate that the 2-min protocol produced more marked bone necrosis than the 1-min protocol. Although our results cannot be entirely extrapolated to clinical practice, they contribute to the understanding of the behavior of bone tissue submitted to different cycles of liquid nitrogen freezing and may serve as a basis for new studies.
\end{abstract}

Key words: Cryosurgery. Osteonecrosis. Jaw diseases.

\section{INTRODUCTION}

The prefix "cryo" is derived from the Greek word "kryos", which means cold. Cryosurgery is a method that uses low temperatures for local tissue destruction ${ }^{4}$. The locally lethal effects of this method are the result of cell dehydration and the formation of intracellular ice crystal, causing direct cytotoxic injury and secondary vascular ischemia $11,12,13,25$.

The maxillomandibular complex is prone to a variety of lesions that, although benign, might be locally aggressive $9,10,22,24$. Doubts exist regarding the best therapeutic approach in these cases, since conservative management has been associated with high rates of recurrence, whereas radical treatment, although resulting in cure in most cases, may cause severe esthetic-functional impairment ${ }^{21}$. In this respect, adjuvant therapies, such as cryosurgery, have been combined with conservative modalities in order to reduce the rate of recurrence without increasing morbidity ${ }^{5,21,23}$.

Animal models investigating the effects of cryosurgery on bone tissue have generally been used for descriptive purposes and have only evaluated morphological aspects $3,7,8,14,15,19,20,27$. A broad review of the English-language literature revealed no standardized studies that histomorphometrically analyzed the effects of cryotherapy on bone tissue. The aim of the present study was to evaluate the effects of liquid nitrogen cryosurgery on the femoral 
diaphysis of rats using a standardized experimental model and histomorphometric parameters.

\section{MATERIAL AND METHODS}

\section{Animals}

Forty-two male Wistar rats aged 16 weeks $(360-460 \mathrm{~g})$, randomly chosen from the Central Animal House of the Federal University of Ceará, Fortaleza, CE, Brazil, were used in this study. The animals were housed in separate cages on a 12-24 $\mathrm{h}$ light/dark cycle at $23-25^{\circ} \mathrm{C}$, with free access to food and water. The experiments were reviewed by the Animal Care and Use Committee of the same institution (protocol number: 6/2006) and were conducted according to recommended guidelines on animal experimentation.

\section{Cryosurgery protocols}

Freezing was carried out in a CRY-AC ${ }^{\circledR}-3$ cryostat (model \#B-700, Brymill, imported from CRY$A C^{\circledR}$, Brazil) using liquid nitrogen as coolant and a closed cryoprobe with a flat surface measuring $1 \mathrm{~mm}$ in diameter. The animals were divided into two groups according to the cryosurgery protocol used: group A was submitted to three 1-min freeze cycles intercalated with periods of $5 \mathrm{~min}$ of passive thawing, for a total freeze time of 3
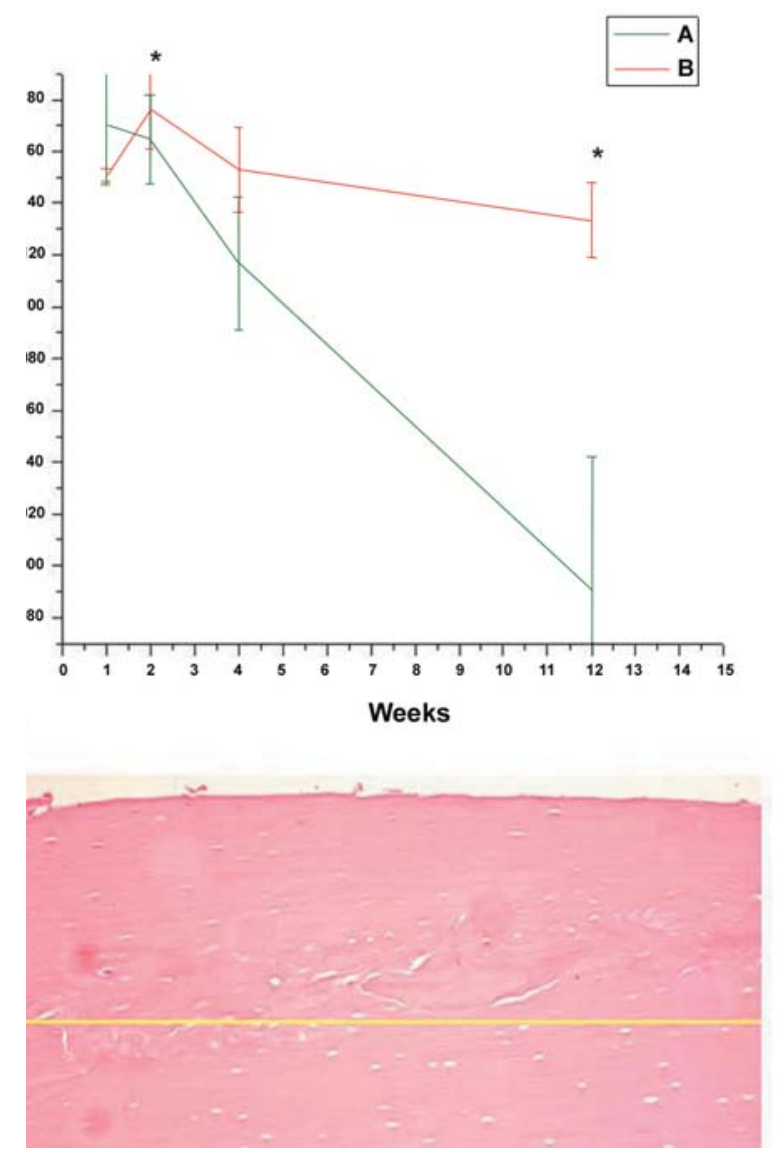

min; group B was submitted to three 2-min freeze cycles intercalated with periods of 5 min of passive thawing, for a total freeze time of $6 \mathrm{~min}$.

\section{Surgical procedures}

The rats were anesthetized by intraperitoneal injection of $2.5 \%$ tribromoethane $(0.1 \mathrm{mg} / 100 \mathrm{~g}$ body weight). Next, a $1.5-\mathrm{cm}$ linear incision was made along the lateral aspect of the right thigh from the proximal femur. The anterior thigh muscles were cut longitudinally and the femoral diaphysis was exposed. The joint ligaments were released and the tip of the closed probe was positioned on the bone surface $1 \mathrm{~cm}$ from the head of the femur. Retractors were carefully positioned to avoid damage to adjacent soft tissues during the freezing process. After cryosurgery, the muscles were returned to their initial position, and the soft tissues and skin were sutured. The animals were allowed to recover and were placed in individual cages. No drugs were administered postoperatively.

\section{Histologic preparation}

Groups of rats were sacrificed by cervical dislocation 1, 2, 4 and 12 weeks after surgery. The right femurs were removed, fixed by immersion in $10 \%$ neutral buffered formaldehyde for $48 \mathrm{~h}$ at room temperature, and then decalcified in $5 \%$ acid nitric
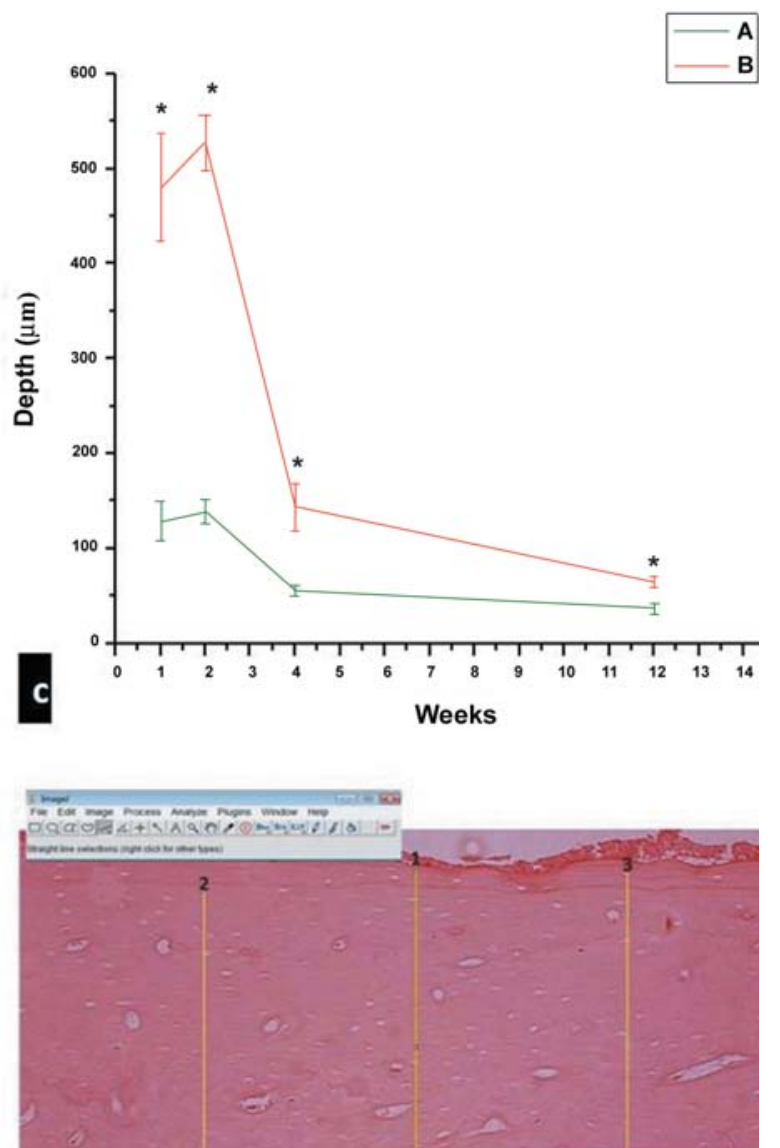

Figure 1- Comparison of the linear extent $(a, b)$ and depth $(c, d)$ of bone necrosis between the 1-min $(A)$ and 2-min (B) cryosurgery protocols according to experimental week (mean $\pm S D$ ). ${ }^{*} p<0.05$ (comparison between protocols $A$ and $B$ ) 
for 10 days. After decalcification, the specimens were dehydrated in a graded ethanol series, placed in xylene, and embedded in paraffin in such a way that the femur could be sectioned in the sagittal plane. The site of cryoapplication was determined by positioning a millimeter rule $1 \mathrm{~cm}$ from the femoral head. Sections ( $4-\mu \mathrm{m}$-thick) were cut sequentially from the lateral border to the site of cryoapplication and stained with hematoxylin and eosin.

\section{Histomorphometric analysis}

For histomorphometric analysis, the data were processed using the Image J version software 1.43 $\mathrm{s}$ (National Institutes of Health, Bethesda, MD, USA; available from http://rsbweb.nih.gov/ij/). The most central stained section that corresponded to the site of cryoapplication was selected in each block. The sections were examined under a Leica DMLB light microscope (Leica Microsystems, Nussloch, Germany) connected to a Nikon Alphaphot-2 VS2 digital camera (Nikon, Tokyo, Japan). In each section, all histologic fields were analyzed and saved as digital images. The images were acquired using a 100x objective with a fixed grid and the following parameters were measured: (1) depth of bone necrosis, (2) extent of bone necrosis, (3) number of empty osteocyte lacunae, and (4) number of empty vessel channels. For depth and extent of bone necrosis, pixel values were converted into micrometers using a Neubauer chamber (0.1 $\mathrm{mm} / 0.0025 \mathrm{~mm}^{2}$ ) at the same magnification. In addition, in each histologic field three vertical standardized measurements were made for the assessment of the extent of bone necrosis (Figure 1b), and a horizontal measurement was performed for the evaluation of the depth of bone necrosis (Figure 1d). Empty osteocytes and vessel channels are expressed as a numerical ratio, with the calculation of the proportion between empty and total lacunae ("empty" and "full") and between empty and total vessel channels (Figure 2a).
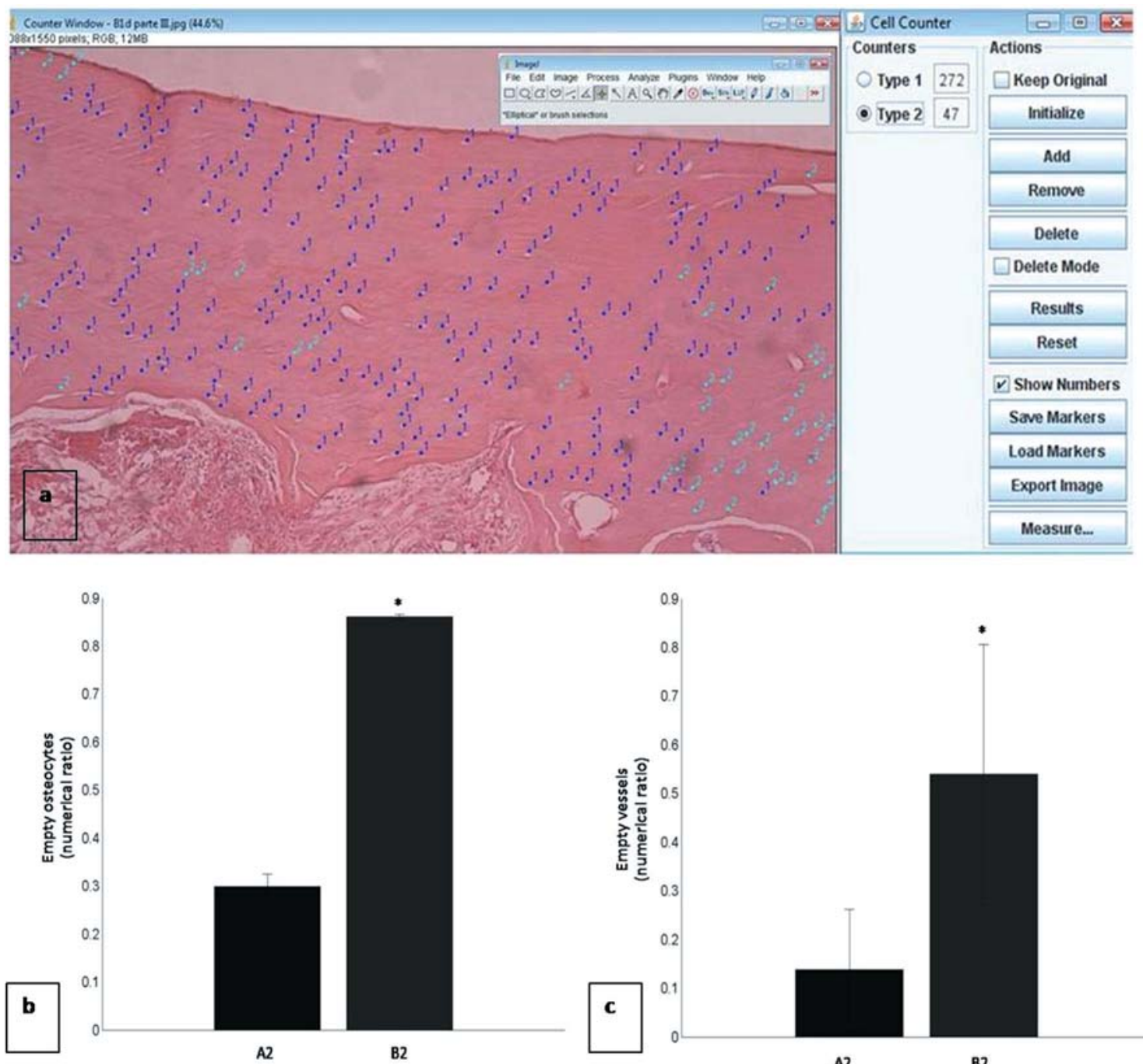

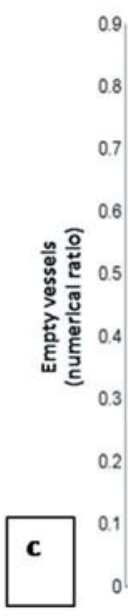

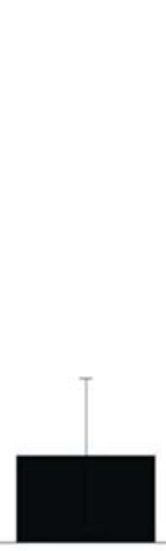

A2

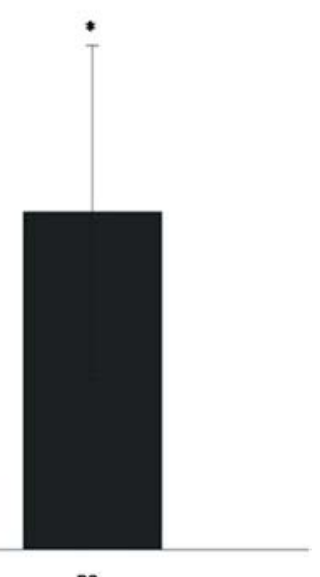

B2

Figure 2- Image J software (a) used for the determination of the number of empty (type 1) and full (type 2) osteocytes/vessel channels. Numerical ratio between empty osteocytes (b)/vessel channels (c) and total osteocytes/vessel channels according to experimental week (mean $\pm S D$ ). A2, 1-min protocol; B2, 2-min protocol. " $p<0.05$ (comparison between protocols A and B) 


\section{Statistical analysis}

All measurements were made blindly, with the examiner being unaware whether the 1 -min or 2-min protocol had been applied. Median, quartiles and minimum and maximum values were calculated for the variables studied. Statistical analysis was performed using the Origin 8.0 statistical program (Micronal Software, Northampton, MA, USA). The results were compared using the Kruskal-Wallis test and Dunn's post-hoc test was applied to groups in which more than two samples differed significantly. A level of significance of $5 \%$ was adopted $(p<0.05)$.

\section{RESULTS}

\section{One-minute protocol}

The absolute peak extent of bone necrosis $(2087.094 \mu \mathrm{m})$ was observed in the second week of the experiment. Comparison showed a significant difference $(p<0.05)$ between experimental weeks, except between the first and fourth week. Pairwise comparison between weeks of observation showed a significant difference $(p<0.05)$ in mean depth of bone necrosis per histologic field for most combinations, except between the first and second week. In addition, peak necrosis depth was $124.509 \mu \mathrm{m}$ in the second week. A marked percentage of both empty osteocytes (Figure 2b) and vessel channels (Figure $2 \mathrm{c}$ ) was observed in the second week, with mean ratios of 0.31 and 0.34 , respectively.

\section{Two-minute protocol}

The peak extent of bone necrosis was 12046.426 $\mu \mathrm{m}$ and was observed in the second week of the experiment. A significant difference in necrosis extent was only observed between the first and second week $(p<0.05)$ (Figure 1a). Regarding the depth of bone necrosis, a significant difference was observed between all weeks of this study $(p<0.05)$ (Figure 1c). In addition, a mean depth of 436.424 $\mu \mathrm{m}$ was found in the second week. Necrosis of osteocytes (Figure 2b) and vessel channels (Figure 2c) was mainly observed in the second week, with mean ratios of 0.64 and 0.71 , respectively.

\section{DISCUSSION}

In an attempt to reduce or even to prevent esthetic-functional complications associated with radical treatment of benign aggressive jaw diseases, alternative therapies such as the application of tissue fixatives (Carnoy solution) and cryogens (liquefied gauzes at a temperature below $0^{\circ} \mathrm{C}$ ) have been combined with conservative treatment ${ }^{1,17,21,23,26}$.

On the basis of morphofunctional phylogenetic investigations ${ }^{18}$, we developed an experimental model using rat femurs for comparative studies in order to extrapolate the clinical use of cryotherapy to human jaw diseases. For standardization of the present method, a closed probe system was chosen since it permits better control of the quantity of tissue involved ${ }^{2}$.

The present study is the first employing histomorphometric parameters for the evaluation of the tissue response to cryosurgery. The Image J software was used since it is a simple, public domain program freely available on the internet, which has also been used for other purposes in dentistry as reported by Demirbas ${ }^{6}(2008)$.

Analysis of the extent of osteonecrosis in animals submitted to the 1-min protocol showed no significant difference in mean values between histologic fields of animals evaluated after the same period of time. However, pairwise comparison of the different observation periods showed a significant difference between practically all periods, except between the first and fourth week. The highest mean total extent of necrosis was observed in the second week.

In contrast to the 1-min protocol, a significant difference in the mean extent of bone necrosis between histologic fields was observed in the second week for animals submitted to the 2-min protocol. Similarly, the highest mean extent of necrosis was observed in the second week compared to the other groups, but the difference was only significant when compared to the first week of observation.

Comparison of the protocols of liquid nitrogen cryosurgery showed a similar mean extent of necrosis between weeks, except for a significant difference between the second and $12^{\text {th }}$ week.

Another important parameter for the quantitative analysis of morphologic alterations resulting from cryotherapy is the percentage of necrotic osteocytes. Using the 1-min protocol, a significant difference in this parameter was observed in the second week when compared to the other weeks. The same was noted for the other experimental protocol. Comparison of the two protocols showed a significantly higher mean percentage of necrotic osteocytes for the 2-min protocol compared to the 1-min protocol, corresponding to peak osteocyte necrosis in the present study.

In addition to the quantification of osteocyte necrosis, significant differences in the devitalization of vessel channels were also observed between groups of the two protocols. As observed for the previous morphologic analysis, a significantly higher percentage of necrotic channels were noted in the second week of observation compared to the remaining weeks, with peak vascular necrosis also occurring in the second week as observed for osteocyte necrosis. In contrast, the lowest percentage of necrotic cortical vessels was observed 
in the last week of observation after cryotherapy, corresponding to peak vascularization in the present study.

The depth of necrosis in animals is generally evaluated by thermographic imaging, a procedure in which thermal needles connected to the target tissues monitor the local temperature field during the cryosurgical procedure ${ }^{14}$. Using this approach, Bradley and Fisher $^{3}$ (1975) evaluated different methods of freezing bone in $2-\mathrm{cm}$ wide surgical cavities of dry porcine mandibles. The authors observed that the use of nitrogen spray resulted in complete bone penetration, including the opposite cortex, after $5 \mathrm{~min}$. In contrast, the use of closed probes resulted in a small area of cortical bone after $10 \mathrm{~min}$ where the cellular temperature reached a lethal level. However, these studies provided only immediate and in vitro results, whereas an in vivo longitudinal analysis using qualitative and quantitative parameters was performed in the present study.

We also observed differences in the depth of osteonecrosis between histologic fields, between weeks of observation and between the two cryosurgery protocols. Using the 1-min protocol, a significant difference in mean depth of bone necrosis between histologic fields was only observed in the group sacrificed after 1 week, with the observation of greater homogeneity in the band of necrotic cortical bone in the other groups. The highest mean necrosis depth was observed in the second week of evaluation, with this value being numerically similar to that obtained in the first week and significantly higher than those obtained in the remaining weeks using the same protocol.

In contrast to the 1-min protocol, when using the 2-min protocol, a difference in mean necrosis depth was observed not only for the one week group but also for the two week group. These groups also showed greater heterogeneity of the cellular effects of cryotherapy, with specific fields showing a greater necrosis depth. Mean necrosis depth was significantly higher in animals sacrificed after 2 weeks, similar to the findings obtained with the 1-min protocol.

In the present study, mean peak necrosis was significantly higher in the second week of observation using the 2-min liquid nitrogen cryotherapy protocol compared to the 1-min protocol. This finding agrees with Kuylenstierna ${ }^{15}$ (1980) who studied rabbit mandibles and observed marked bone necrosis in the second week of evaluation. In contrast, in a histologic study on rabbit femurs, Keijser ${ }^{14}$ (1999) demonstrated a clear demarcation of osteonecrosis one week after cryosurgery, similar to the findings obtained with the 1-min protocol of the present study.

Clinically, bone fractures after cryotherapy have mainly been reported for ameloblastoma involving the upper jaw bones, a benign but locally aggressive odontogenic tumor ${ }^{1,5,7,16,17,21}$. Despite the absence of pathological fractures in the present study, we suggest that the marked bone necrosis observed especially in the second experimental week may contribute to the occurrence of this complication, a fact supporting the need for careful postoperative follow-up.

\section{CONCLUSIONS}

The findings of the present study indicate that the 2-min protocol produced more accentuated bone necrosis than the 1-min protocol. Although our results cannot be entirely extrapolated to clinical practice, they contribute to the understanding of the behavior of bone tissue submitted to different cycles of liquid nitrogen freezing and may serve as a basis for new studies. Experimental data are important for the understanding of the mechanisms underlying the effects of cryosurgery.

\section{REFERENCES}

1- Blanas N, Freund B, Schwartz M, Forst IM. Systematic review of the treatment and prognosis of the odontogenic keratocyst. Oral Surg Oral Med Oral Pathol Oral Radiol Endod. 2000;90:553-8. 2- Bradley PF. Modern trends in cryosurgery of bone in the maxillofacial region. Int J Oral Surg. 1978;7:405-15.

3- Bradley PF, Fisher AD. The cryosurgery of bone: an experimental and clinical assessment. Br J Oral Surg. 1975;13:111-27.

4- Courage GR, Huebsch RF. Cold therapy revisited. J Am Dent Assoc. 1971;83:1070-3

5- Curi MM, Dib LL, Pinto DS. Management of solid ameloblastoma of the jaws with liquid nitrogen spray cryosurgery. Oral Surg Oral Med Oral Pathol Oral Radiol Endod. 1997;84:339-44.

6- Demirbaş AK, Ergün S, Güneri $P$, Aktener BO, Boyacioğlu H. Mandibular bone changes in sickle cell anemia: fractal analysis. Oral Surg Oral Med Oral Pathol Oral Radiol Endod. 2008;106:e41-8. 7- Emmings FG, Neiders ME, Greene GW Jr, Koepf SW, Gage AA. Freezing the mandible without excision. J Oral Surg. 1966;24:14555.

8- Fisher AD, Williams DF, Bradley PF. The effect of cryosurgery on the strength of bone. Br J Oral Surg. 1978;15:215-22.

9- Flórez-Moreno GA, Henao-Ruiz H, Santa-Sáenz DH, Castañeda-Peláez DA, Tobón-Arroyave SI. Cytomorphometric and immunohistochemical comparasion between central and peripheral giant cell lesions of the jaws. Oral Surg Oral Med Oral Pathol Oral Radiol Endod. 2008;105:625-32.

10- González-Alva P, Tanaka A, Oku Y, Yoshizawa D, Itoh $S$, Sakashita $H$, et al. Keratocystic odontogenic tumour: a retrospective study of 183 cases. J Oral Sci. 2008;50:205-12.

11- Hafron J, Kaouk JH. Cryosurgical ablation of renal cell carcinoma. Cancer Control. 2007;14:211-7.

12- Han B, Bischof JC. Direct cell injury associated with eutectic crystallization during freezing. Cryobiology. 2004;48:8-21.

13- Hoffman NE, Bischof JC. The cryobiology of cryosurgical injury. Urology. 2002;60:40-9.

14- Keijser LC, Schreuder HW, Buma P, Weinans H, Veth RP. Cryosurgery in long bones: an experimental study of necrosis and revitalization in rabbits. Arch Orthop Trauma Surg. 1999;119:4404. 
15- Kuylenstierna R, Anniko M, Lundquist PG, Nathanson A. Experimental cryosurgery on bone: a light and electron microscopical investigation. Criobiology. 1980;17:563-70.

16- Marciani RD, Trodahl JN, Suckiel MJ, Dubick MN. Cryotherapy in the treatment of ameloblastoma of the mandible: report of cases. J Oral Surg. 1977;35:289-95.

17- Morini P, Sanvitto LC, Dias WB. Criotherapy in ameloblastomas. Odontol Mod. 1982;9:28-38.

18- Najjar TA, Kahn D. Comparative study of healing and remodeling in various bones. J Oral Surg. 1977;35:375-9.

19- Natiella JR, Meenaghan MA, Rosa RA, Bessette RW, Gage AW. Cryosurgery of major and minor salivary gland: a light microscopic evaluation in the Rhesus monkey. J Oral Pathol. 1979;8:237-46. 20- Popken F, Land M, Erberich H, Bosse M, König DP, Eysel P. The use of a new miniature cryoprobe for ablation of bone tissue: in vivo assessment of the probe and application of the method to bone in a sheep model. BMC Surg. 2003;3:3.

21- Salmassy DA, Pogrel MA. Liquid nitrogen cryosurgery and immediate bone grafting in the management of aggressive primary jaw lesions. J Oral Maxillofac Surg. 1995;53:784-90.
22- Sannomiya EK, Silva JV, Brito AA, Saez DM, Angelieri F, Dalben GS. Surgical planning for resection of an ameloblastoma and reconstruction of the mandible using a selective laser sintering 3D biomodel. Oral Surg Oral Med Oral Pathol Oral Radiol Endod. 2008;106:36-40.

23- Schmidt BL, Pogrel MA. Neurosensory changes after liquid nitrogen cryotherapy. J Oral Maxillofacial Surg. 2004;62:1183-7. 24- Sivakumar G, Kavitha B, Saraswathi TR, Sivapathasundharam B. Odontogenic myxoma of maxilla. Indian J Dent Res. 2008; 19:62-5.

25- Theodorescu D. Cancer cryotherapy: evolution and biology. Rev Urol. 2004;6:S9-S19.

26- Webb DJ, Brockbank J. Treatment of the odontogenic keratocyst by combined enucleation and cryosurgery. Int J Oral Surg. 1984;13:506-10.

27- Whittaker DK. Repeat freeze cycles in cryosurgery of oral tissues. Br Dent J. 1975;139:459-65. 